\title{
An extragalactic supernebula confined by gravity
}

Turner, J. L., * Beck, S. C., † Crosthwaite, L. P. , *† Larkin, J. E. , ${ }^{*}$ McLean, I. S., * \& Meier, D. S. * $\S$

*Department of Physics and Astronomy, UCLA, Los Angeles CA 90049-1562 USA

†Department of Physics and Astronomy, University of Tel Aviv, Ramat Aviv, Israel

†Astute Networks, 16868 Via Del Campo Court,Suite 200, San Diego CA 92127

§Department of Astronomy, 1002 West Green Street, University of Illinois, Champaign, IL 61801 USA

Little is known about the origins of the giant star clusters known as globular clusters. How can hundreds of thousands of stars form simultaneously in a volume only a few light years across - the distance of the sun to its nearest neighbor? Radiation pressure and winds from luminous young stars should disperse the starforming gas and disrupt the formation of the cluster. Globular clusters in our Galaxy cannot provide answers; they are billions of years old. Here we report the measurement of infrared hydrogen recombination lines from a young, forming super star cluster in the dwarf galaxy, NGC 5253. The lines arise in gas heated by a cluster of an estimated million stars, so young that it is still enshrouded in gas and dust, hidden from optical view. We verify that the cluster contains 4000-6000 massive, hot " $O$ " stars. Our discovery that the gases within the cluster are bound by gravity may explain why these windy and luminous $O$ stars have not yet blown away the gases to allow the cluster to emerge from its birth cocoon. Young clusters 


\section{in "starbursting" galaxies in the local and distant universe may be similarly gravitationally confined and cloaked from view.}

NGC 5253 is host to hundreds of large star clusters ${ }^{1}$, including dozens of extremely bright, young super star clusters ${ }^{2}$ (SSCs). Only a few million years old ${ }^{3,4}$, these clusters are found in the central "starburst" 4 region of the nearby (3.8 Mpc) galaxy. Subarcsecond radio and infrared imaging reveal ${ }^{5,6,7}$ a bright "supernebula" within the starburst, optically invisible, probably powered by a young super star cluster. ${ }^{8,9}$ The luminosity required to ionize the supernebula is $0.8-1.2 \times 10^{9} \mathrm{~L}_{\odot}$ within a $1-2 \mathrm{pc}{ }^{8}$ region, perhaps the most concentrated star-forming luminosity known.

To verify the nature of the supernebula and to study its dynamics, we observed the Brackett $\square$ and $\square$ recombination lines of hydrogen at $4.05 \square \mathrm{m}$ and $2.17 \square \mathrm{m}$ using the NIRSPEC $^{10}$ on the Keck Telescope on 11 March 2000. Spectra were taken through a 0.579 " x 24 " slit at a spectral resolution of R 25,000, or $\sim 12 \mathrm{~km} / \mathrm{s}$. SCAM, a 256x256 array camera within NIRSPEC, simultaneously imaged the slit location on the galaxy at K-band (2.2 $\square \mathrm{m})$, allowing us to pinpoint where the spectra were taken. Seeing was $0.55 "-0.8 "$

The $2.2 \square \mathrm{m}$ broadband image of NGC 5253 reveals hundreds of bright infrared star clusters, shown in Figures 1 and 2. From their optical colors, Calzetti et al. ${ }^{3}$ and Tremonti et al. ${ }^{4}$ estimate that these SSCs are only $\sim 2-50 \mathrm{Myr}$ in age. We find that the brightest infrared source does not coincide with any of the optical clusters. The brightest $2.2 \square \mathrm{m}$ source is offset by $\sim 0.3 " \pm 0.1$ " to the northwest of the youngest ${ }^{3}$ optical source. This visually-obscured $\mathrm{K}$ band source is the location of the strong Brackett line emission that we observe from the supernebula. 
Figure 2 shows the image of the slit position with the strongest Brackett line emission and the corresponding Brackett $\square$ echellogram. Line plots of both Brackett lines are shown in Figure 3. Continuum-subtracted line fluxes are $\mathrm{S}_{\mathrm{Br}}{ }_{\square}^{\text {obs }}=3.4 \pm 1 \times 10^{-16} \mathrm{~W} \mathrm{~m}^{-}$ ${ }^{2}$ and $\mathrm{S}_{\mathrm{Br}}{ }^{\text {obs }}=3.9 \pm 1 \times 10^{-17} \mathrm{~W} \mathrm{~m}^{-2}$ for a region within $\sim 3$ " of the continuum peak. For comparison, Kawara, Nishida \& Phillips ${ }^{11}$ measure $\mathrm{S}_{\mathrm{Br} \square}{ }^{\text {obs }}=7.0 \pm 1 \times 10^{-16} \mathrm{~W} \mathrm{~m}^{-2}$ and $\mathrm{S}_{\mathrm{Br}}$ ${ }_{\square}^{\text {obs }}=1.5 \pm 1 \times 10^{-16} \mathrm{~W} \mathrm{~m}^{-2}$ in a $10^{\prime \prime} \times 20$ " aperture; as much as half of their Brackett $\square$ flux may be contributed by an unsubtracted continuum. The large aperture flux at $\mathrm{K}$ band appears to be dominated by stellar emission, while much of the L' flux is from nebular dust. Our photometry and mapping confirm that most, if not all, of the Brackett $\square$ and at least $30 \%$ of the Brackett $\square$ emission in NGC 5253 emerges from a source coincident with the brightest $\mathrm{K}$ band continuum source.

Having both Brackett lines allows us to correct the line fluxes for extinction and obtain a good estimate of the true, unextincted recombination line flux, and thus the total ionizing flux. For an intrinsic $\mathrm{S}_{\mathrm{Br} \square}$ obs $/ \mathrm{S}_{\mathrm{Br} \square}$ obs flux ratio of $2.8^{13}$, a temperature of $12000 \mathrm{~K}$ ${ }^{14}$, and a Rieke \& Lebofsky ${ }^{15}$ extinction law, we obtain extinctions of $\mathrm{A}_{\square}=0.8$ magnitudes at $4.05 \square \mathrm{m}$ and $A_{\square}=2.0$ magnitudes at $2.17 \square \mathrm{m}$. The corresponding optical extinction is $A_{v}=18$ magnitudes. Optical Balmer recombination lines give $A_{v}=3^{14}$. The apparent contradiction between the low optical extinction and higher infrared extinction is resolved if the extinction is high and internal to the nebula. The extinction-corrected Brackett line fluxes are $\mathrm{S}_{\mathrm{Br} \square}{ }^{\text {obs }}=7.1 \times 10^{-16} \mathrm{~W} \mathrm{~m}^{-2}$ and $\mathrm{S}_{\mathrm{Br} \square}$ obs $=2.5 \times 10^{-16} \mathrm{~W} \mathrm{~m}^{-2}$ for the inner $\sim 1.5$ ". These fluxes predict a $15 \mathrm{GHz}$ free-free flux of $24 \pm 8 \mathrm{mJy}$ : the observed 15 GHz flux in this region, corrected for opacity ${ }^{9}$, is $24 \pm 3$ mJy. ${ }^{6}$ The Lyman continuum rate required to maintain the ionization of the supernebula is $\mathrm{N}_{\mathrm{Lyc}}=4 \pm 1 \times 10^{52} \mathrm{~s}^{-1}$, equivalent to $4000 \mathrm{O} 7$ stars. This agrees with $\mathrm{cm}$-wave and mm-wave free-free fluxes ${ }^{6,8,9}$ and radio recombination lines ${ }^{16}$ (Owens Valley mm-wave fluxes ${ }^{9}$ give a value of 600007 stars from a larger aperture). Both the excellent agreement of the Brackett line fluxes and 
radio fluxes and the observed compactness of the emission argue that the Brackett line emission arises from the radio "supernebula".

The velocity information presented here is new; these spectra probe the dynamics of the nebula at high spatial and spectral resolution. The Brackett line profiles were fit with Gaussians, with line centroids at $\mathrm{v}_{\mathrm{LSR}}=377 \pm 2 \mathrm{~km} \mathrm{~s}^{-1}$ and full widths half maxima of $76 \pm 1 \mathrm{~km} \mathrm{~s}^{-1}$, consistent with $\mathrm{H} \square^{.17}$ and radio recombination lines ${ }^{16}$. For gas at 12,000 $\mathrm{K}$, this linewidth is supersonic. Supersonic gas motions are expected in nebulae: in addition to nebular expansion there is also the interaction of the nebular gas with winds from the massive stars in the cluster. For comparison, compact HII regions in our Galaxy around small groups of $\mathrm{O}$ stars have linewidths of up to $64 \mathrm{~km} / \mathrm{s}^{18}$; nebulae around individual O stars in the Wolf-Rayet phase can have linewidths of 50-200 km/s. ${ }^{19}$

The first implication of the linewidth of $75 \mathrm{~km} / \mathrm{s}$ for the "supernebula" is that if the nebula were actually expanding at the implied speed of $\sim 38 \mathrm{~km} / \mathrm{s}$, then the mean radius of $\sim 0.7 \mathrm{pc}^{8}$ of the nebula implies an implausibly short dynamical age of 7000 years. Dynamical ages of compact HII regions in our Galaxy are generally too short to explain the numbers of observed nebulae ${ }^{20}$. It is thought that confinement mechanisms such as the pressure of dense molecular clouds are responsible for the retardation of their expansion. This could lengthen the lifetime of the supernebula phase, if there were molecular gas nearby, which is as yet undetected ${ }^{9}$.

The more unusual implication of the linewidth is that while supersonic, these nebular lines are remarkably narrow given the size of the nebula and the high luminosity of its embedded star cluster. Brackett line, radio, and infrared fluxes all require $\mathrm{L}_{\mathrm{OB}} \sim 0.8-$ $1.2 \times 10^{9} \mathrm{~L}_{\odot}$ for excitation of the nebula. For a cluster with a Salpeter initial mass function (IMF) and a lower mass cutoff of $1 \mathrm{M}_{\odot}$, the mass in stars corresponding to this luminosity is $5-7 \times 10^{5} \mathrm{M}_{\odot}$; if the IMF extends down to stars less than $1 \mathrm{M}_{\odot}$, the cluster 
mass may reach $10^{6} \mathrm{M}_{\odot}$. The size of the radio nebula is well-determined, and should be the same as the Brackett size since both emissions are proportional to $\int \mathrm{n}_{\mathrm{e}}{ }^{2} \mathrm{dl}$; VLA images show that the nebula is $0.9 \mathrm{pc} \times 1.8 \mathrm{pc}$ in size $(0.05 " \mathrm{x} 0.1 ", \pm 0.02 ")^{8}$. We assume that the star cluster exciting the nebula lies within the supernebula or else the implied excitation luminosity would be higher than the total observed IRAS luminosity of the entire galaxy, $1.8 \times 10^{9} \mathrm{~L}_{\odot}{ }^{7}$. For a radius of $0.5-0.9 \mathrm{pc}$, the escape velocity is $\sim 25-30 \mathrm{~km} \mathrm{~s}^{-}$ ${ }^{1}$ for a cluster of $\mathrm{O}$ stars alone; $\mathrm{v}_{\mathrm{esc}} \sim 50-70 \mathrm{~km} \mathrm{~s}^{-1}$ for an IMF cutoff at $1 \mathrm{M}_{\odot}$; and $\mathrm{v}_{\mathrm{esc}}$ $\sim 85-110 \mathrm{~km} \mathrm{~s}^{-1}$ for a cluster IMF extending below $1 \mathrm{M}_{\odot}$. Gravity must play a significant role in the dynamics of this nebula, slowing its expansion if not halting it. Virial linewidths are only slightly smaller than $\mathrm{v}_{\mathrm{esc}}$, so the nebula could actually be in gravitational equilibrium.

If the "supernebula" stage of super star cluster formation should prove to be common and long-lived, it may have implications for the detection of Lyman $\square$ and other ultraviolet lines in star-forming galaxies. There is evidence that Ly $\square$ is detectable primarily in galaxies in which the potential absorbing gas is Doppler-shifted out of the path of the Ly $\square$ photons. ${ }^{21}$ This would be most likely in galaxies with superwinds and superbubbles, phenomena which can be caused by large concentrations of hot and windy $\mathrm{O}$ stars. ${ }^{22}$ If a significant fraction of the lifetime of the ionizing phase of a super star cluster is spent in a confined, extincted state such as the supernebula, it is likely that these winds will develop only late in the formation process of the cluster, perhaps at the occurrence of the first supernovae. By that time the ionizing flux of the cluster is already declining. Super star clusters may be an important mode of star formation in the early universe. Ly $\square$ searches for primeval galaxies may therefore fall far short of detecting the true star formation rate, as suggested by observations. ${ }^{21,23,24,25}$ 
To put the supernebula in context we can compare it to a more traditional nebula, 30 Doradus in the Large Magellanic Cloud. 30 Doradus is a large, luminous nebula ionized by an optically visible star cluster with an estimated age of $<10 \mathrm{Myr},{ }^{26} * * 150-200 \mathrm{pc}$ in extent and with a gas density of $20-100 \mathrm{~cm}^{-3} .{ }^{27} 30$ Doradus is 200 times larger and 1001000 times less dense than the supernebula in NGC 5253, and its exciting star cluster R136 is ten to 100 times less massive than the cluster in NGC $5253 .{ }^{28}$ The escape velocity for 30 Doradus is less than the thermal sound speed of $10 \mathrm{~km} / \mathrm{sec}$ so its gas motions are determined by winds and turbulence rather than gravity. ${ }^{29}$ How will the supernebula in NGC 5253 free itself from gravitational bondage and evolve into an extended, diffuse nebula like 30 Doradus? That will probably depend on the evolution of its underlying star cluster.

1. Caldwell, N., \& Phillips, M. M. Star formation in NGC 5253. Astrophys. J. 338, 789803 (1989).

2. Gorjian, V. WFPC2 imaging of the starburst galaxy NGC 5253. Astron. J., 1120, 18861893 (1996).

3. Calzetti, D. et al. Dust and recent star formation in the core of NGC 5253. Astron. J., 114, 1834-1849 (1997).

4. Tremonti, C. A., Calzetti, D., Leitherer, C., \& Heckman, T. M. Star formation in the field and clusters of NGC 5253. Astrophys. J., 555, 322-337 (2001).

5. Beck. S. C., Turner, J. L., Ho, P. T. P., Kelly, D., \& Lacy, J. H. The central star cluster of the star-forming dwarf galaxy NGC 5253. Astrophys. J., 457, 610-615 (1996).

6. Turner, J. L., Ho, P. T. P., \& Beck, S. C. The radio properties of NGC 5253 and its unusual HII regions. Astron. J., 116, 1212-1220 (1998). 
7. Gorjian, V., Turner, J. L., \& Beck, S. C. Infrared emission from the radio supernebula in NGC 5253: a proto-globular cluster? Astrophys. J, 554, L29-L32 (2001).

8. Turner, J. L., Beck, S. C., \& Ho, P. T. P. The radio supernebula in NGC 5253. Astrophys. J, 532, L109-112 (2000).

9. Meier, D. S., Turner, J. L., \& Beck, S. C. Molecular gas and star formation in NGC 5253 Revisited. Astron. J., 124, 877-885 (2002).

10. McLean, I. S., et al., Performance and results with the NIRSPEC echelle spectrograph on the Keck II Telescope. Proc. Spie 4008, 1048-1055 (2000)

11. Kawara, K., Nishida, M., \& Phillips, M. M. Brackett alpha and gamma observations of starburst and Seyfert galaxies. Astrophys. J., 337, 230-235 (1989).

12. Moorwood, A. F. M., \& Glass, I. S. Infrared Emission and Star Formation in NGC 5253. Astron. Astrophys. 115, 84-89 (1982).

13. Storey, P. J. \& Hummer, D. G. Recombination line intensities for hydrogenic atoms. IV. - Total recombination coefficients and machine-readable tables for $\mathrm{Z}=1$ to 8 . Mon. Not. R. Astron. Soc, 272, 41-48 (1995).

14. Walsh, J. R., \& Roy, J.-R. Optical spectroscopic and abundance mapping of the amorphous galaxy NGC 5253. Mon. Not. R. Astron. Soc, 239, 297-324 (1989).

15. Rieke, G. H., \& Lebofsky, M. J. The interstellar extinction law for 1 to 13 microns. Astrophys. J., 288, 618-621 (1985). 
16. Mohan, N. R., Anantharamaiah, K. R., \& Goss, W. M. Very Large Array observations of the H92 $\square$ Line from NGC 5253 and Henize 2-10: Ionized Gas around Super Star Clusters. Astrophys. J., 557, 659-670 (2001).

17. Martin, C. L., \& Kennicutt, R. C., Jr. Soft X-ray emission from NGC 5253 and the ionized interstellar medium. Astrophys. J., 447, 171-183 (1995).

18. De Pree, C. G., Wilner, D. J., Goss, W. M., Welch, W. J., \& McGrath, E. Ultracompact HII regions in W49N at 500 AU scales: shells, winds, and the water maser source. Astrophys. J., 540, 308-315 (2000).

19. Chu, Y.H. Ring nebulae around massive stars throughout the HR diagram. IAU Symposium 212, van der Hucht, K. A., Herrero, A., \& Esteban, C., eds. in press (2002)

20. Wood, D. O. S., and Churchwell, E. Massive stars embedded in molecular clouds: their population and distribution in the Galaxy. Astrophys. J., 340, 265-272 (1989).

21. Kunth, D., Mas-Hesse, J. M., Terlevich, E., Terlevich, R., Lequeux, J., \& Fall, S. M. HST study of Lyman-slpha emission in star-forming galaxies: the effect of neutral gas flows. Astron. Astrophys., 334, 11-20.

22. Tenorio-Tagle, G., Silich, S. A., Kunth, D., Terlevich, E., \& Terlevich, R. The evolution of superbubbles and the detection of Lya in star-forming galaxies. Mon. Not. R. Astron. Soc., 309, 332-342.

23. Meier, D. L., \& Terlevich, R. Extragalactic HII regions in the UV. Implications for primeval galaxies. Astrophys. J., 246, L109-L113 (1981).

24. Hartmann, L., Huchra, J. P., \& Geller, M. J. How to find galaxies at high redshift. Astrophys. J., 287, 487-491 (1984). 
25. Steidel, C. C., Giavalisco, M., Pettini, M., Dickinson, M., Adelberger, K. L.

Spectroscopic confirmation of a population of normal star-forming galaxies at redshifts z>3. Astrophys. J., 462, L17-L21 (1996).

26. Walborn, N. R., \& Barbá, R. H. The new 30 Doradus. New Views of the Magellanic

Clouds, ed. Y.-H. Chu, N. Suntzeff, J. Hesser, \& D. Bohlender, 213 (1999).

27. Mills, B. Y., Turtle, A. J., Watkinson, A., A radio model of the 30 Doradus region. Mon. Not. R. Astron. Soc., 185, 263-276 (1978)

28. Kennicutt, R. C., Jr., \& Chu, Y.-H., Giant HII Regions and the Formation of Populous Clusters. Astron. J., 95, 720-730 (1988)

29. Melnick, J., Tenorio-Tagle, G., \& Terlevich, R. Supersonic gas motion in extragalactic HII regions. Mon. Not. R. Astron. Soc., 302, 677-683 (1999)

The authors thank Tiffany Glassman and Eric Greisen for assistance with the data and Michael Jura for helpful discussions. This research is supported by NSF grant AST00-71276, and a grant from the Israel Academy Center for Multi-Wavelength Astronomy.

Correspondence and requests for materials should be addressed to J.T. (e-mail: turner@astro.ucla.edu).

Figure 1. Infrared-optical color view of the young super star clusters of NGC 5253. The $\square=2.2 \square \mathrm{m}$ image (red channel) was constructed from SCAM images made during the night. The infrared image is combined with an optical image from the Hubble Space Telescope (blue and green channels). Seeing was 0.7-0.8" for the infrared image; the HST image was convolved to match. The brightest K-band source appears as an extended red source to the north of the 
dust lane. A Gaussian fit to this source gives a size (FWHM) of 1.10" x 0.9”, p.a. $36^{\circ}$. From smaller clusters we estimate the point spread function for the SCAM image to be $0.75 " \pm 0.2 "$. If the K-band source is Gaussian, this would imply a source size of $\sim 0.8^{\prime \prime} \times 0.5^{\prime \prime}$; along p.a. $40^{\circ}$. This size is uncertain because of variable seeing. The entire SCAM image is 46 " square. The orientation of the image is east to the left, north up.

Figure 2. Echellogram and slit position for Brackett spectra of the supernebula. The 46" 446 " SCAM image shows the position of the 0.579 " $x 24$ " slit on the brightest K-band source in NGC 5253. In the inset is the Brackett $\square$ echellogram, with frequency/velocity running horizontally and the spatial dimension vertically. The full slit length is 24 ", but only 6 " is shown in the inset. The orientation of the image is such that north is at an angle of $-43^{\circ}$ (clockwise) from vertical. The Brackett line emission is less than 1.3" in spatial extent on the echellogram, the same as the standard star. Spectra taken at eight other positions showed weak or no emission

Figure 3. Spectra of $\mathrm{Br} \square$ and $\mathrm{Br} \square$ in NGC 5253. The spectra were integrated over the central 3" centered on the brightest infrared continuum source. Each grating setting was followed by a calibration A star. On/off slit SCAM images of the standard indicate that $50 \%$ of the light entered the slit. Since the Brackett line emission has spatial extent comparable to the standard star, $\sim 6-9$ pixels $(0.8 "-1.3 ")$, calibration using the standard should automatically correct for this effect. We estimate an uncertainty of $30 \%$ in the line fluxes due to variability in seeing. Near-infrared continuum emission from the brightest $\mathrm{K}$ band source is so strong that it can be seen even in these highly dispersed spectra. We measure continuum fluxes of $186 \pm 60 \mathrm{mJy}$ and $12 \pm 4 \mathrm{mJy}$ at L' and K, 
respectively, as compared to the $144 \mathrm{mJy}$ and $20 \mathrm{mJy}$ fluxes at in these bands observed by Moorwood \& Glass ${ }^{12}$ with 8-9" apertures.

${ }^{* *}$ Note added after publication: The age of the R136 cluster is significantly less than the $10 \mathrm{Myr}$ quoted in the published article, as corrected here. Our thanks to Nolan Walborn for catching this error. Conclusions are unaffected. 




Figure 1

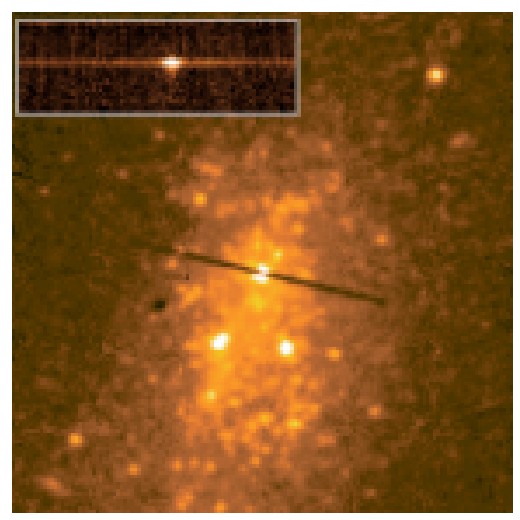

Figure 2 


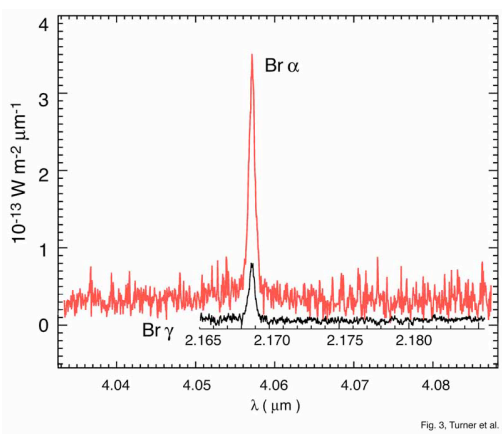

